\title{
Analyzing Human Gait and Posture by Combining Feature Selection and Kernel Methods
}

\author{
Albert Samà a,1, Cecilio Angulo ${ }^{\mathrm{b}, *}$, Diego Pardo ${ }^{\mathrm{a}}$, \\ Andreu Català ${ }^{\mathrm{b}}$, Joan Cabestany ${ }^{\mathrm{b}}$ \\ CETpD - Technical Research Centre for Dependency Care and Autonomous \\ Living. Vilanova i la Geltrú, Barcelona, Spain \\ ${ }^{\text {a } F H C S A A ~-~ S a n t ~ A n t o n i ~ A b a t ~ H o s p i t a l ~}{ }^{3}$ \\ ${ }^{\mathrm{b}} U P C$ - Technical University of Catalonia ${ }^{2}$
}

\begin{abstract}
This paper evaluates a set of computational algorithms for the automatic estimation of human postures and gait properties from signals provided by an inertial body sensor. The use of a single sensor device imposes limitations for the automatic estimation of relevant properties, like step length and gait velocity, as well as for the detection of standard postures like sitting or standing. Moreover, the exact location and orientation of the sensor is also a common restriction that is relaxed in this study.

Based on accelerations provided by a sensor, known as the ' $9 \times 2$ ', three approaches are presented extracting kinematic information from the user motion and posture. Firstly, a two-phases procedure implementing feature extraction and Support Vector Machine based classification for daily living activity monitoring is presented. Secondly, Support Vector Regression is applied on heuristically extracted features for the automatic computation of spatiotemporal properties during gait. Finally, sensor information is interpreted as an observation of a particular trajectory of the human gait dynamical system, from which a reconstruction space is obtained, and then transformed using standard principal components analysis, finally Support Vector Regression is used for prediction.
\end{abstract}

Daily living Activities are detected and spatiotemporal parameters of human gait are estimated using methods sharing a common structure based on feature extraction and kernel methods. The approaches presented are susceptible to be used for medical purposes.

Key words: Human gait and posture detection, inertial body sensor, Kernel methods application, Time series analysis

PACS: 
2 One of the consequences of chronic diseases and strokes is the limitation of the motion capacity and a straightforward lack of physical activity, having a direct impact on quality of life of the patient. By extracting spatiotemporal parameters from human gait and posture, medical treatments would count with valuable additional information, allowing a better diagnose and treatment assessment for diseases like Parkinson's [1], diabetes [2], and for the early detection of other conditions like risk of falling, avoiding possible hip break episodes and its consequences in elderly people [3].

Usual instruments to supervise patients mobility are based on the subjective perceptions of an observer or the use of large and expensive measurement equipment like posturometers or walkway systems [4]. Moreover, during the last decade several advances have been developed on wearable systems based on accelerometry for the automatic extraction of spatiotemporal gait parameters [27] and daily activity monitoring [28]. Compactness and objectiveness of inertial based devices allow the development of truly ambulatory systems predicting and detecting gait anomalies in real-time, overcoming the need of questionnaires [5] and clinical trials, where users may act differently from real life conditioned by the environment (e.g., being observed) and other uncontrolled variables like lack of memory of the patients.

The use of inertial sensors to extract this information has been successfully applied in diverse studies, e.g., [6]. Nevertheless, available systems for the reliable ambulatory extraction of spatiotemporal gait parameters usually require the use of several devices [7] and often carrying a bunch of wires along the body communicating devices $[8,9]$. Moreover, recently developed wireless ambulatory systems $[9,10]$ still need more than one device in order to extract features like step size, stride length, and step velocity from human gait using gyroscopes tied at legs. Wearing these devices on the legs during daily life activity seems a drawback, leaving the application scope of this method to clinical environments. In the case of accelerometers, they are usually positioned at the dorsal side of the trunk, near the region of the L3 vertebra of the subject, since it is the Center of Mass (CoM) location. In this position, 3D CoM ac-

* Corresponding Author.

Email address: cecilio.angulo@upc.edu (Cecilio Angulo).

$U R L$ : www. upcnet.es/ upc15838 (Cecilio Angulo).

1 Author is granted by the Spanish Ministry of Science and Innovation project MoMoPa (PI08/90756)

2 The authors would like thank Carlos Pérez-López, Marc Torrent-Poch, and Jordi Parera-Giró for their technical support.

3 The authors would like thank Alejandro Rodríguez-Molinero for his clinical support. 
celeration, velocity and displacement can be estimated [10,11]. However, our studies on usability indicated that this position is not practical when sitting or performing some daily physical activities. To the best of our knowledge, there is no any user-friendly wearable device / location that patients may use outside the hospital.

A measurement system composed by a single device would cover the need of an ambulatory solution easy to be worn during daily life. This system imposes a challenge for the extraction of reliable information from the limited signals obtained. This paper study the use of one of such simple systems, motivated by the high impact that it will have on the end-users acceptability. Other approaches using just one device [11], are sensitive to the precise location and adjustment of the sensor on the patient: lumbar zone, chest or lateral hip. Some of them inclusive requires a non-intuitive location (foot, knee, ankle), forcing the user to modify natural motions during sitting, standing and laying postures and transitions. Besides, works on ambulatory activity monitoring using a single sensor rely on the off line processing of logged data. The purpose of our research is to analyze human gait and posture using features extracted from signals provided online by a small-sized wearable sensor module located in the patient's waist. Therefore, this system can be used everywhere during daily life avoiding the need of special infrastructure. The measurement system employed in this study is briefly described in Section 2 where a comparison with other devices is also presented.

This work is based on two results, the first one is oriented to demonstrate that the system can be used to detect diverse human postures, thus, using kernel based algorithms, the system offers detection properties similar to those of already commercially available systems. Secondly, kernel methods are used to extract gait spatiotemporal properties from accelerometry data.

The posture detection and gait properties estimation approaches may be discriminated as follows: (i) A two-phases procedure implementing feature extraction from raw acceleration signals and Support Vector Machine (SVM) based classification; (ii) The use of Support Vector Regression (SVR) on heuristically extracted features from acceleration signals for step length and velocity estimation and (iii) an approach based on the assumption that sensor information encapsules information of an unknown dynamical system resulted during the human gait, standard principal component analysis and SVR completes this spatiotemporal properties estimation.

The remaining of the paper is organized as follows. Section 2 reviews other accelerometry systems used to analyze human motion, it also presents a comparison with the accelerometry system used in this study. Section 3 presents the approach to identify among 5 common motion activities. Section 4 describes a regression approach to estimate step length and velocity from acceleration 
signals collected from the subject's waist. Section 5 tackles the same problem but using an approach based on intrinsic properties of a hidden dynamical system. Finally, Section 6 concludes the paper with some remarks and comments about future research.

\section{System Overview}

First a review of the features of some accelerometry based systems is presented, then the system used for this study is described and compared with other devices.

\subsection{Accelerometry Based Systems for Human Motion Systems}

Lately, detection and classification of human daily living activity have received wide attention from the research community. Besides the sensor used in this study, so-called ' $9 \mathrm{x} 2$ ', there are already several commercial physical activity monitors that manage to detect several activities: Shimmer [29] is a small wireless wearable sensor that can also record and wirelessly transmit physiological and kinematic data in real-time. Its small size, however, constraints battery duration to 3-4 hours when using a $50 \mathrm{~Hz}$ sampling rate. Xsense MTi [31] is a system containing gyroscopes, accelerometers and magnetometers. The internal low-power digital signal processor runs a real-time sensor fusion algorithm providing drift-free 3D orientation data. Xsense MTw [31] sends data using RF communication technology, however battery duration is reduced from 18.5 to 3.5 hours. The mcroberts DynaPort MiniMod [32] supports applications where a subject wears the sensor at the lower back for a longer period of time under free living conditions and it is able to analyze the patient's quantity of movement.

Other specialized platforms exist, having battery life as main feature, like activPAL [33]. It identifies and classifies individual's free-living activity like sitting, standing and walking. Data can be collected during 10 days using a very low sampling rate, and no on-line process can be implemented. Physilog, developed at EPFL, [8] has not wireless data transmission. MicroStrain 3DMGX1 [35] considers 9 axes of measurement, but it presents the same restrictions that Xsens MTi. Finally, Activity Monitor [33] is an IMU, worn in the wrist, developed to measure physical activity. It is endowed with RF for wireless communication.

Special attention should be presented to the commercial platform MiniSun IDEEA (Intelligent Device for Energy Expenditure and Activity) [34] specifi- 
cally designed to measure movement, it may compute duration, frequency and intensity of diverse types of human physical activity (PA). The working principle of IDEEA is the constantly monitoring of the body and limb motions through five sensors attached to the chest, thighs, and feet. Data are then downloaded to a computer for off-line analysis at the end of each test. For the calibration of IDEEA, the subject was asked to sit in an upright position with feet and thighs parallel to the floor and the upper body in a vertical position. Calibration takes 5 seconds, this process ensures a maximal deviation of 15 degrees in each direction. Although it detects locomotion well (such as walk-ing or running), activities involving mainly arm motion, such as rowing, swinging a ball or bat, operating a vacuum cleaner, etc., would not be correctly identified.

These examples demonstrates that despite that human activity is already successfully identified using commercially available devices, it is either employing several sensors on the patient's body or extracting data to be processed ofline, preventing the use of its outputs in real-time applications like tele-care, automatic infusion of drugs or ambient intelligence integration. Restricting the number of devices in the system and demanding on-line detection and extraction imposes challenges for the technical and algorithmic approaches.

\section{2 '9×2' System Description}

The inertial system is a single unit device. All the electronic components plus a Li-on battery $(1000 \mathrm{mAh})$ are encapsuled in a $78 \times 37 \times 10 \mathrm{~mm}$ black case. It weights $125 g$ (battery included). The prototype also includes a wall battery charger. Fig. 1 shows the prototype and its corresponding $\mu \mathrm{SD}$ card.

Internally, the system includes the classical elements of an Inertial Measurement Unit (IMU) as well as a system dedicated to the battery control and energy consumption optimization. The status of both, the battery level and the main application process, is shown to the user using a very simple user interface comprised of three LEDs (Light Emission Diodes). A switch allows the user to interact with the device at any time. Figure 1 shows the device.

Table 1 shows a technical comparison of the analyzed commercial platforms. Presented information for each platform is its sampling rate, battery life, dimensions, processing capacity, datalog function, wireless communication, and sensors included (accelerometers, gyroscopes and magnetometers). 


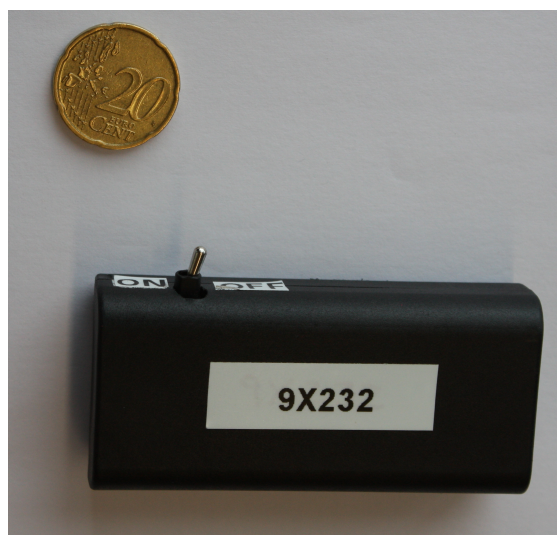

Fig. 1. Sensor prototype

\begin{tabular}{|c|c|c|c|c|c|}
\hline Name & $\mathrm{Hz}$ & hours & $\operatorname{dim}$ & CPU.Data.Wi & Ac.Gy.Ma \\
\hline CETpD-UPC 9x2 & 200 & 18 & $75 \times 37 \times 21$ & Y.Y.Y & Y.Y.Y \\
\hline Xsens MTi & 120 & 9.4 & $53 \times 38 \times 20$ & N.N.N & Y.Y.Y \\
\hline Xsens MTw & 150 & 3.5 & $58 \times 34 \times 14$ & N.N.Y & Y.Y.Y \\
\hline Shimmer 9DoF & 50 & $3-4$ & $53 \times 32 \times 19$ & N.N.N & Y.N.N \\
\hline Shimmer WSP & - & 3.5 & $50 \times 25 \times 12$ & N.Y.Y & Y.N.N \\
\hline miniSun IDEEA & 32 & 60 & $70 \times 55 \times 18$ & ?.Y.N & Y.N.N \\
\hline mcroberts DynaPort & 100 & 47 & $83 \times 51 \times 9$ & N.Y.N & Y.N.N \\
\hline PAL ActivPAL & 20 & 240 & $53 \times 35 \times 7$ & N.Y.N & Y.N.N \\
\hline EPFL Physilog & 200 & 14 & $61 \times 50 \times 18$ & N.Y.N & Y.Y.N \\
\hline Activity Monitor & 50 & 24 & $46 \times 36 \times 15$ & N.N.Y & Y.N.N \\
\hline MicroStrain 3DM-GX1 & 100 & 15.4 & $90 \times 64 \times 25$ & $? . N . N$ & Y.Y.Y \\
\hline
\end{tabular}

Table 1

IMU-based physical activity monitors. Presented information for each platform is its sampling rate $(\mathrm{Hz})$, battery life (hours), dimensions (dim), processing capacity (CPU), datalog function (Data), wireless communication (Wi), and sensors included, i.e., accelerometers (A), gyroscopes(Gy) and magnetometers (Ma)

\section{Daily Living Activities Identification}

As a first approach to demonstrate the system properties and the type of on-line algorithms that are studied, this section presents an experience of treatment of acceleration data provided by the ' $9 \times 2$ '. Identification of human activities is completed using kernel methods. 


\subsection{Methodology}

A test group of subjects was employed to collect data from the sensor while performing activities. The group was confirmed by 6 healthy subjects with no mobility limitations and aged $38.17 \pm 12.6$. Data was collected while subjects perform the following sequence of activities: Stay steady in vertical position, walk about 4 meters, sit down on a chair, stay sit down for a few seconds, stand up, walk, sit down again to finally stay sit down. This sequence was repeated 3 times for each subject and experiments were video recorded to enable labeling of activities.

\subsection{Activities Analysis}

Signals and video information from the following activities were manually isolated and analyzed,

(1) Standing up. This activity lasts for 1 to 2.5 seconds with differentiated phases: forward bending, active raising, passive raising and downward bending. Timing and magnitude between phases may determine several pathological characteristics.

(2) Sitting down. This action also ranges from 1 to 2.5 seconds. It is similar to inverse of standing up, these pairs of signals being the most similar in the group of activities.

(3) Transition Movement. Due to high similarity between sit down and stand up activities, an auxiliary control state has been created to classify activities different from stand or sit signals. It can be viewed as an activity occurring just before (after) sitting down (standing up).

(4) Walking activity. Step duration varies between 0.5 and 1.5 seconds, classifiers are trained to look for a complete walking episode instead of focusing on individual steps.

(5) Steady activity. Acceleration signals for this activity are simple and predictable, but it occurs on unbounded time windows. Steady activity is similar when user is sitting or standing.

\subsection{Signal Processing}

The duration of the activities to be classified ranges from 0.5 seconds, for walking, up to 2.4 seconds, for the stand up activity. Since raw data is obtained sampling at $50 \mathrm{~Hz}$, the use of windows of 120 samples ensures the capture of the longest type of activity. 
- Orientation angles: Earth gravity allows to calculate orientation for the sensor device. Formulation works fine in static movement conditions. Impact of low centripetal accelerations is not important [13], nevertheless impacts or large accelerated movements incorporate error.

$$
\theta(k)=\arctan \left(a_{x}(k), \sqrt{a_{y}^{2}(k)+a_{z}^{2}(k)}\right), \phi(k)=\arctan \left(a_{y}(k), a_{z}(k)\right)
$$

- Vertical $a_{V}(k)$ and forward $a_{F}(k)$ components: Accelerations in the inertial reference (fix frame) can be computed from the mobile reference using the orientation angles. Features values are robust to the measuring device orientation:

$$
\begin{gathered}
a_{F}(k)=\cos (\theta(k)) a_{x}(k)+\sin (\theta(k)) a_{z}(k) \\
a_{V}(k)=-\cos (\phi(k)) \sin (\theta(k)) a_{x}(k)+\sin (\phi(k)) a_{y}(k)+\cos (\theta(k))\left(a_{z}(k)+g\right)
\end{gathered}
$$

190

- Energy expenditure indicators: acceleration signals are used to calculate the integral of absolute value (IAA) and the integral of magnitude (IAV) ${ }^{4}$ :

$$
I A A=\sum_{k}\left(\left|a_{x}(k)\right|+\left|a_{y}(k)\right|+\left|a_{z}(k)\right|\right), I A V=\sum_{k} r(k) d t
$$

- Increments in the acceleration module,

$$
\Delta r(k)=r(k)-r(k-1) .
$$

Frequency-based features can be obtained by performing the Fast Fourier Transform (FFT) on the acceleration signals, however this approach is out of the scope of this research due to the high processing time demanded when implementing its computation within the microprocessor of the sensor.

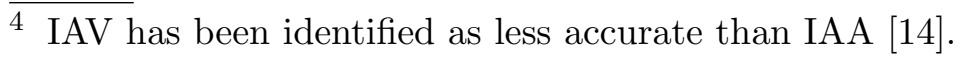




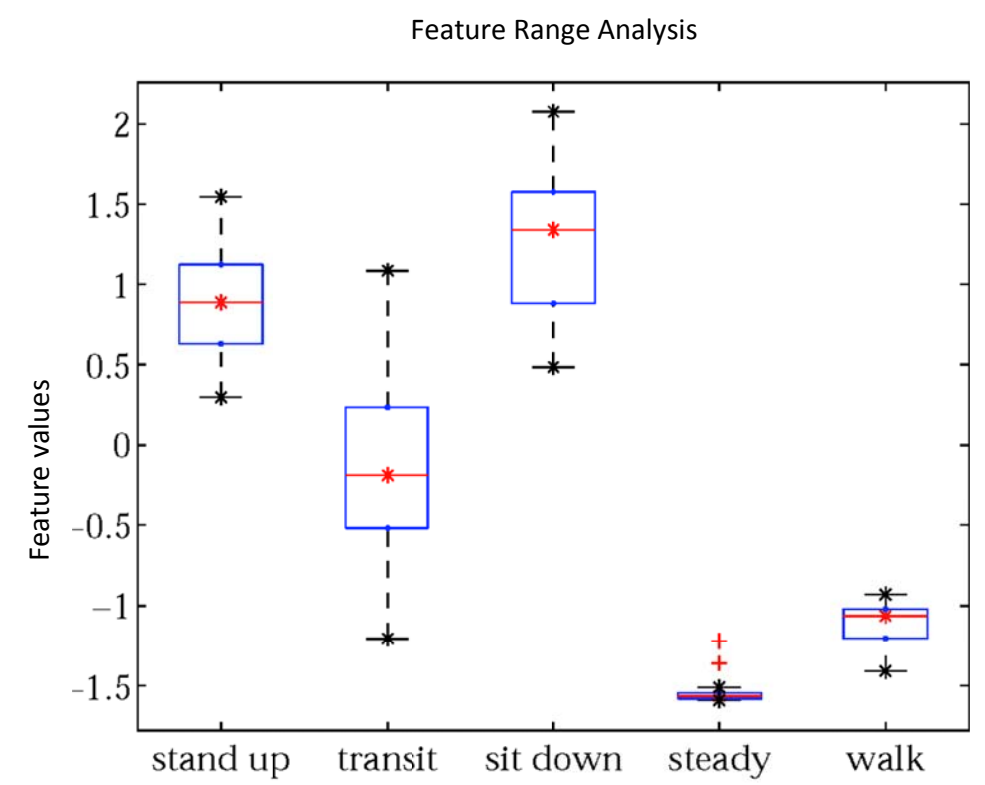

Fig. 2. Intervals for the 5 activities based on 'range $\theta$ ' feature.

\subsection{Feature Selection}

Standard statistical properties of the previously defined signals, i.e., mean, max, min, range, standard deviation, entropy, etc, conform a huge set of features characterizing the behavior of the signal. This has already been proved to be useful in similar SVM-based works [15]. These features capture relevant information about the motion within a given time-window, enabling the process of data stream, i.e., input data for the classification algorithm containing relevant information of the correspondent window allows a batch process every half-size window.

A data base with observations of the activities provided by different users is generated. Fig 2 presents a sample feature ('range $\theta$ ') using boxplots, which bounds are defined on the $25 \%$ and the $75 \%$ percentile. All features are normalized so relevance and performance for each feature can be compared [16].

Whiskers to bound intervals are defined as the most extreme data value within $\frac{3}{2} \cdot I Q R$, where $I Q R$ is the interquartile range of the sample. Data outside the whiskers are considered as outliers.

Giving a pair of intervals $(A, B)$, let us define a separation value between them as,

$$
d=\max \left\{\begin{array}{l}
\min A-\max B \\
\min B-\max A
\end{array}\right.
$$


Separation between intervals is used to select discriminant features, i.e., interclass distances are used to rank features similarly to [30]. A symmetric matrix $D_{f} \in \mathbb{R}^{5 \times 5}$ for any given feature $f$ represents its separation to the remaining features. Note that the main diagonal in $D_{f}$ is composed by zeros. Whether different activities are represented by non overlapping data ranges, then separation values are positive, therefore this feature classify the pair of implied activities by itself. Negative values of separation are interpreted as overlapping among classes. Nevertheless, separability among classes is improved using several features.

The number of classes that a given feature can discriminate is given by the number of rows containing distances greater than a given threshold. This threshold may be fixed to zero if no overlapping among classes is desired.

Three indicators were tested to incrementally select the best set of features:

(1) The number of pair of activities that a single feature discriminates using a given threshold, i.e. the number of positive values for each distance matrix $D_{f}$. Given that the main diagonal is null, the maximum number of possible positive values is 20 . Nevertheless, not a single feature demonstrated to be a discriminant for all classes.

(2) The sum of distances between classes, a value representing how different is a feature for each class or activity. Since features are normalized and negative values for overlapping activities are considered, values from -20 to +20 can be expected.

(3) The sum of positive distances between classes. In this case only not overlapping intervals are considered, being a particular case of the previous indicator. Here, values range from 0 to +20 .

\subsubsection{Detection Results and Analysis}

Using the indicators presented above, features were ordered according to its class separability degree. The length of the vector of features was not restricted in order to check the method accuracy with different vector lengths. Five one-versus-rest SVM classifiers in a multi-class structure were trained with Gaussian kernel, inputs being assigned to the voted class. Whether no positive votes exist or more than one class voted, then no label is assigned. A 10-fold cross-validation procedure, with 3 repetitions, was performed for each length of the feature vector, ranging from 1 to 30 features.

Figure 3 shows the maximum accuracy reached by any of the three feature vector for each possible length. The best result was obtained when the sum of positive distance between classes was used as indicator and the length of the feature vector is 7 . Its correspondent accuracy is $91.06 \%$, while the selected features are: 'std $a_{x}(k)^{\prime}, ' \min a_{x}(k)$ ', 'std $a_{V}(k)$ ', 'std $a_{F}(k)$ ', 'min $a_{F}(k)$ ','std 


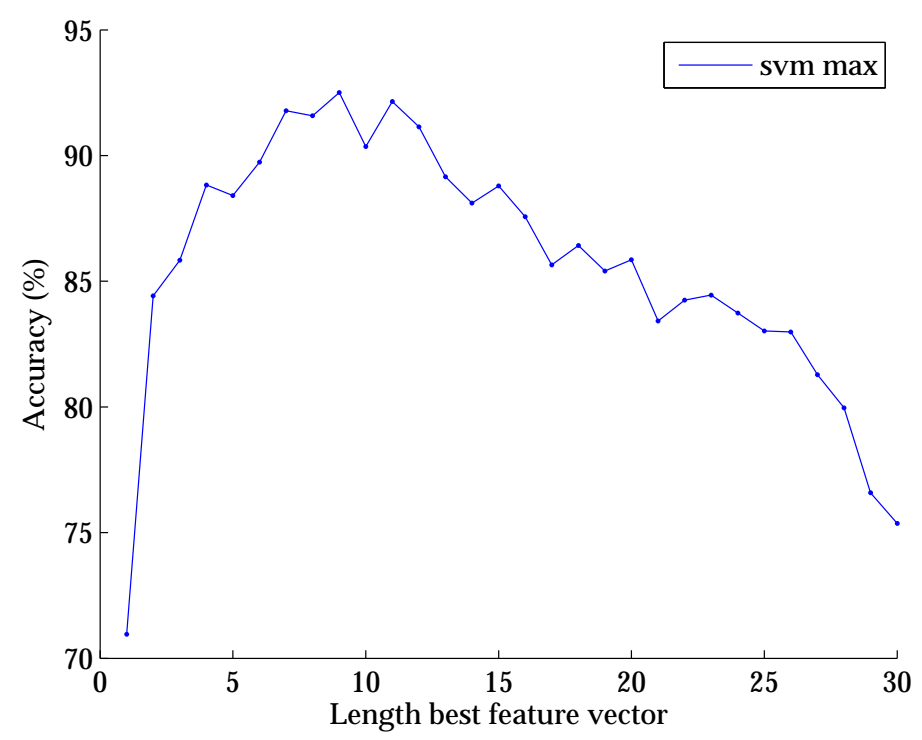

Fig. 3. Accuracy results versus number of features used.

$\theta(k)$ ' and 'range $\theta(k)$ ', where 'std' stands for standard deviation. Table 2 contains the confusion matrix for this experiment. It can be noted how stand up / sit down and steady / walk are two groups of activities perfectly discriminated by the classifier. Stand up and sit down activities are sometimes labeled as a transition movement. It is used to happen when movement is starting or ending, so it is difficult to the classifier discriminate between actions.

\begin{tabular}{|c|c|c|c|c|c|}
\hline Label & Stand up & Sit down & Steady & Walk & Transition \\
\hline Stand up & 75.56 & 14.44 & 0 & 0 & 10 \\
\hline Sit down & 6.06 & 89.09 & 0 & 0 & 4.85 \\
\hline Steady & 0 & 0 & 93.33 & 6.67 & 0 \\
\hline Walk & 0 & 0 & 2.22 & 95.56 & 2.22 \\
\hline Transition & 1.39 & 1.39 & 0 & 3.06 & 94.17 \\
\hline
\end{tabular}

Table 2

Confusion Matrix (\%) when the third indicator and a feature vector of length seven is considered.

Signal processing takes, in the worst case, less than a half window of samples, so the final classifier identifies activities performed with a 1.2 seconds delay. Instead of using raw data as input variable to the kernel method, the use of features to represent the behavior of the acceleration speeds up the training and classification procedures, nevertheless it adds a processing layer which is also time consuming.

Calculating the standard deviation related features requires relative high processing real time efforts that the ' $9 \times 2$ ' can handle. Lower, but still high, accu- 
racy results are achieved using features easier to be calculated. For instance, a $90.03 \%$ accuracy has been obtained when a vector of easy-to-compute features is used.

\section{Estimating Spatiotemporal properties of Human Gait: first ap- proach}

Many motor complications affect spatiotemporal properties of the patients' gait [9]. Length and speed of the steps change due to the increment of the impact of the disease. In this section, a method based on SVM-regression $(\varepsilon-$ SVR) is proposed to extract spatiotemporal properties from signals obtained from the ' $9 \times 2$ ' system. Figure 4 , shows the typical output of the sensor during the human walk. The device is sewed to a belt in order to obtain two main properties: step length and step velocity.

\subsection{Signal Processing for Gait Analysis}

Gait is analyzed based on triaxial accelerations signals from the device when located at any of both lateral sides of the waist. Raw data was low-pass filtered before any analysis using a second-order zero-lag Butterworth filter with a cutoff frequency of $15 \mathrm{~Hz}$, which is enough given that $99 \%$ of energy is contained below $15 \mathrm{~Hz}[19]$.

Accelerations signals obtained from the lateral side of the waist differ from usual signals obtained from the region near L3. Figure 4 shows anteriorposterior acceleration on normal gait obtained by the sensor from both locations. The signal obtained from the L3 region is similar to that reported on the literature [11], where negative peaks of the anterior-posterior acceleration are due to the end of the single support phase and the beginning of double support phase. These peaks are preceded by a positive peak, produced in the feet-floor contact. On the other hand, when the accelerometer is located in the lateral side of the waist, the negative peaks are also observed, but not all the positive peaks appear. Only those contacts generated by the foot of the side where the sensor is located produce positive peaks.

Next, the two-phases methodology for the gait analysis wearing the sensor in this position is presented. 


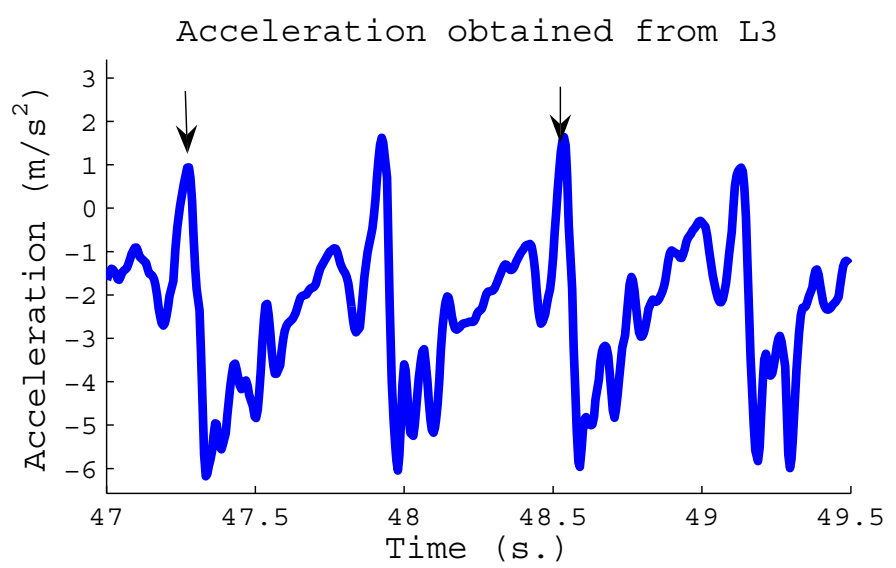

(a) L3 region.

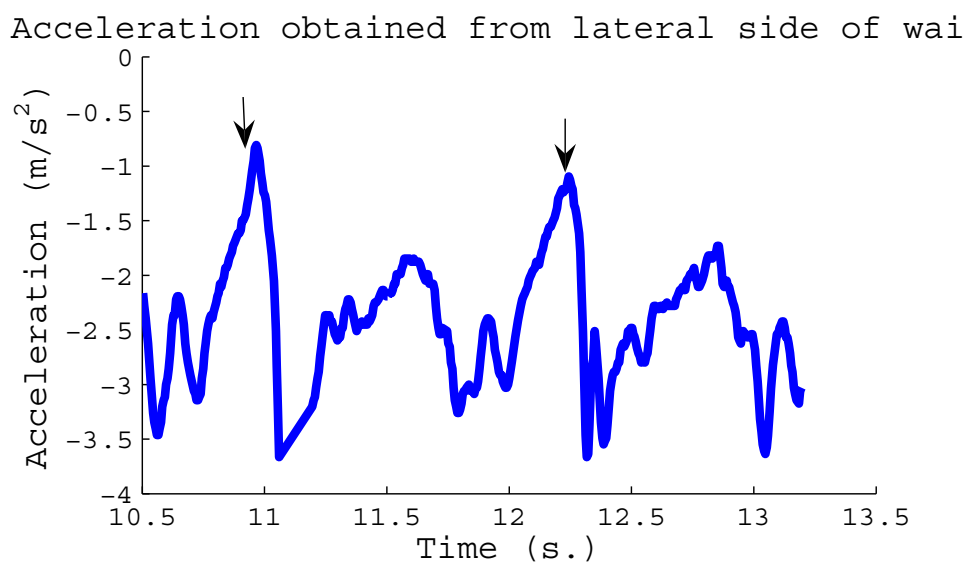

(b) Lateral side.

Fig. 4. Forward acceleration obtained from L3 region, and lateral side of waist.

\subsection{Features Definition}

Biomechanics characteristics of gait allow to automatically identifying steps from tri-axial acceleration signals [6]. Figure 5 shows how segments of acceleration signals related with a step are automatically detected in a pre-processing phase.

Three features are empirically defined for this experimentation based on the acceleration vector $\mathbf{a}(k)$ (Eq. 1) and its module $r(k)$ (Eq. 2) along each timevariant segmented acceleration signals:

(1) the mean of acceleration modules,

$$
\bar{r}_{1, s}=\frac{1}{T_{s}} \sum_{k=k_{s, 0}}^{k_{s, 0}+T_{s}-1} r(k)
$$

where $T_{s}$ is the number of samples during the $s^{\text {th }}$ step, and $k_{s, 0}$ the starting 


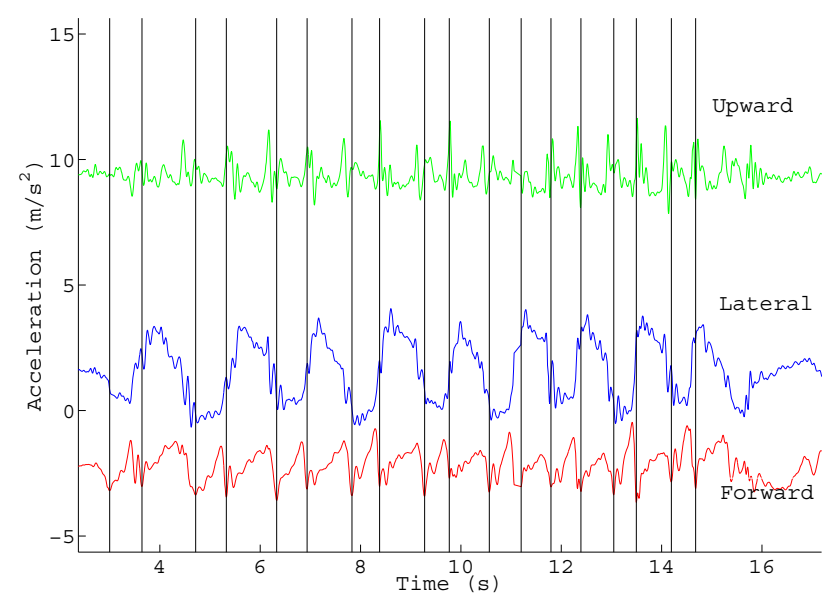

Fig. 5. Automatic step detection.

sample for the $s^{\text {th }}$ segmented step;

(2) the sum of the absolute value of the components,

$$
\bar{r}_{2, s}=\sum_{k=k_{s, 0}}^{k_{s, 0}+T_{s}-1}\left(\left|a_{x}(k)\right|+\left|a_{y}(k)\right|+\left|a_{z}(k)\right|\right)
$$

which is related to the well-know Energy Expenditure Indicator IAA employed in the precedent experiment;

(3) the mean of the absolute values of the time-step increments,

$$
\bar{r}_{3, s}=\frac{1}{T_{s}-1} \sum_{k=k_{s, 0}+1}^{k_{s, 0}+T_{s}-1}|r(k)-r(k-1)|
$$

i.e the mean of the jerk absolute value.

These features are affected by the stride dynamics, for instance, the energy expended on a stride is related to its length and velocity. The faster the step is the higher the acceleration signal values and its increments are, so the mean norm and the mean jerk reflects it.

Additionally, the length and velocity of a given step, measured directly from the experiments, are denoted as $l_{s}$ and $v_{s}$, respectively. The problem at hands can be formulated as find out mappings $f(\cdot)$ and $g(\cdot)$ such that,

$$
l_{s}=f\left(\bar{r}_{1, s}, \bar{r}_{2, s}, \bar{r}_{3, s}\right) \quad, \quad v_{s}=g\left(\bar{r}_{1, s}, \bar{r}_{2, s}, \bar{r}_{3, s}\right)
$$

The $\epsilon$-Support Vector Regression [20] was the kernel method selected in order to extract this relationship from experimental data as it is able to establish non-linear relations between input and output. 
Ten volunteers were asked to walk several times over a plain surface of $6 \mathrm{~m}$. length while a tri-axial accelerometer recorded the measures. As mentioned before, the accelerometer was attached to a belt and it was approximately located at the lateral side of the waist. Its orientation depends on how the belt was worn by the volunteer, thus quite different positions and orientations were used between volunteers. Experiments were recorded by a video camera. Two methods were used to obtain actual step length: footprints and visual markers. Firstly, volunteer's shoe soles where painted and footprints left were measured. Secondly, visual markers distributed every $30 \mathrm{~cm}$. were used to determine by video recordings step lengths. Actual step velocity was obtained by dividing step length by its duration obtained from recordings. These actual values are used as ground truth required to perform regressions against the sensor information. Local ethics committee approved the study, and subjects participation was informed consent. Technical information about the data acquisition procedures and experiments is available in [25].

A $\epsilon$-Support Vector Regression with a cubic polynomial kernel is designed based on the defined features. In order to evaluate its prediction ability, a randomly selected set composed by $80 \%$ of the steps is used to train the $\epsilon$ SVR, and the remaining data is used to establish mean squared error (MSE) rate. Finally, $\epsilon$-SVR is also compared against linear regression on the same training and evaluation sets for each repetition.

Results for step velocity are summarized in Table 4.3. It can be observed that the mean MSE of $\epsilon$-SVR is significantly lower than the error obtained with linear regression. A mean RMSE error of $14.64 \mathrm{~cm} / \mathrm{s}$ is obtained when using $\epsilon$-SVR for predicting new step velocities.

Features used on predicting step length are the same used on step velocity combined with duration time of the step. Results are also summarized in Table 4.3. MSE value for $\epsilon$-SVR is much lower than obtained on linear regression. However, an MSE value of $340.9 \mathrm{~cm}^{2}$ is obtained, which is greater than the obtained for the case of velocity. It means that prediction of the step length is more complex than the estimation of the step velocity using the same set of features.

\section{Human Gait as a Dynamical System}

\section{As a second approach for gait analysis, extending ideas from [21], human gait is} analyzed as a dynamical system (DS), where internal states behave differently 


\section{Table 3}

\begin{tabular}{c|cc}
\hline & Step velocity & Step length \\
\hline Regression & Mean MSE $(\mathrm{cm} / \mathrm{s})$ & Mean MSE $(\mathrm{cm})$ \\
\hline Linear & 301.5 & 605.5 \\
$\epsilon-$ SVR & 214.37 & 340.9 \\
\hline
\end{tabular}

Summary of results for step velocity and step length.

according to the dynamic state of the system. Gait parameters are considered as unknown intrinsic properties of that DS. Acceleration signals are assumed to be a reliable source of information, where the intrinsic system is encoded in form of embedded time-series. Using this dynamical approach, a spectral approximation is followed as in [22], where principal component analysis (PCA) is applied to a conveniently organized set of time series provided by sensor measurements. It allows to discriminate the internal dynamics of the system and correlate it with the actual spatiotemporal properties obtained during gait.

Let us consider human gait as a completely determined, but unknown, dynamical system, $\mathbf{x}_{k+1}=T\left(\mathbf{x}_{k}\right) \in \mathcal{X}$, where $T$ is a unknown deterministic rule. Internal states $\mathbf{x}_{k}$ cannot be directly observed, however a certain measure $s_{k} \in \mathcal{S} \subset \mathbb{R}$ is available, through a device sensor, for instance, which corresponds to the application of certain function $f: \mathcal{X} \rightarrow \mathcal{S}$. A sequence of measures conforms a $m$-tuple, $\mathbf{r}_{k}\left(\mathbf{x}_{k}\right)=\left(s_{k-m+1}, s_{k-m+2}, \ldots, s_{k}\right)=$ $\left(f\left(\mathbf{x}_{k}\right), f\left(T\left(\mathbf{x}_{k}\right)\right), \ldots, f\left(T^{m-1}\left(\mathbf{x}_{k}\right)\right)\right)$, that is said to belong the reconstruction space, i.e., $\mathbf{r}_{k} \in \mathcal{R}$, where $\mathcal{R} \subset \mathbb{R}^{m}$.

The Takens theorem [23] establishes diverse conditions for the reconstruction space $\mathcal{R}$ to be an embedding of $\mathcal{X}$, i.e. to encapsule dynamics of internal states $\mathbf{x}$. A crucial condition is imposed to the size of $\mathbf{r}_{k}$ in order to guarantee the embedding property,

$$
m>2 \cdot \operatorname{dim}(\mathcal{X})
$$

The reconstruction of the state space is obtained from measurements of a triaxial accelerometer attached to one side of the patients' waist ${ }^{5}$. The module of the acceleration vector $r(k)$ (Eq. 2) is used as the $s_{k}$ measure of the current state of the DS. From this measure, the hidden dynamics of the system can be observed using the reconstruction space with the embedding property. Thus, the elements of the reconstruction space corresponding to the $s^{\text {th }}$ step are

5 It will be now assumed the right side as the one of interest, nevertheless it must be noted that the method is invariant to this selection. 
given by the vectors available in the time-series,

$$
\mathbf{r}^{s}\left(\mathbf{x}_{k}\right)=(r(k-m+1), r(k-m+2), \ldots, r(k))^{T} \quad k=k_{s, 0}: k_{s, 0}+T_{s}-m(5)
$$

where $m<T_{s}$. The value of $m$ must satisfy (4) in order to obtain a valid reconstruction space. Nevertheless, as $\operatorname{dim}(\mathcal{X})$ is unknown, a reasonable high value must be selected.

Defining a matrix $\mathbf{R}^{s}$, an arrangement of the $\mathbf{r}^{s}\left(\mathbf{x}_{k}\right)$ vectors, is a reconstruction of the internal dynamics for the $s$-th step. From now on, notation is simplified,

$$
\mathbf{R}^{\mathbf{s}}=\left(\begin{array}{llll}
\mathbf{r}_{0} & \mathbf{r}_{1} & \cdots & \mathbf{r}_{n_{s}}
\end{array}\right)^{T},
$$

thus, the $i$-th row in $\mathbf{R}^{\mathbf{s}}$ corresponds to a point in the representation space that represents the state of the system $\mathbf{x}$ in the time-step $k$. The whole matrix represents a state trajectory of the states of the body dynamics $\left(\mathbf{x}_{0}, \ldots \mathbf{x}_{n}\right)$ during the $s^{\text {th }}$ step.

Since reconstruction of state space is based on module measures, the method is insensitive to the orientation of the device.

\subsection{Feature Selection}

The size of the reconstruction space $\mathcal{R}$ is determined by the chosen dimension $m$, which should be large enough for leading to a space with capacity to capture the system dynamics. At the same time, using a large number of sensor data, i.e., a large time-series, leads to a large representation space with the valuable information spread along the columns in (6).

The use of principal component analysis theory (PCA) transforms the representation matrix $\mathbf{R}$, a huge database of time-series provided by sensor signals, through a transformation matrix $\mathbf{B} \in \mathbb{R}^{m \times m}$, so that relevant information of the DS behavior is concentrated in first latent variables,

$$
\mathbf{Y}=\mathbf{R B}
$$

i.e., the columns of $\mathbf{Y}$, namely $\mathbf{y}_{j}, j=0, \ldots, m$. Moreover, each row of $\mathbf{Y}$ still corresponds to a given time-step of the state space.

Only the first latent variables, containing most part of information, are employed, then, discarded latent variables are considered to contain only noise. Distinguishing between relevant or noisily variables is performed by observing its contribution: noisily variables hardly contribute in comparison to the 


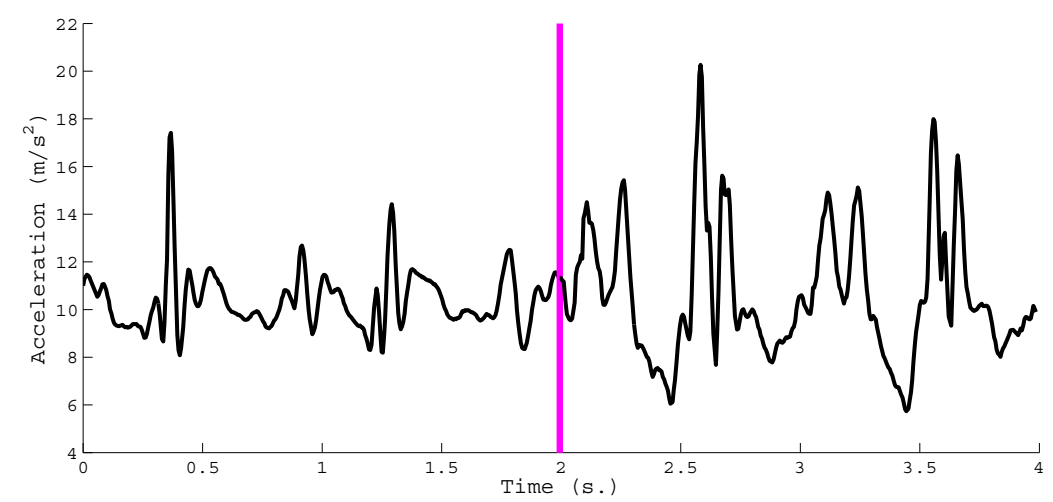

(a) Acceleration Magnitude (raw data)

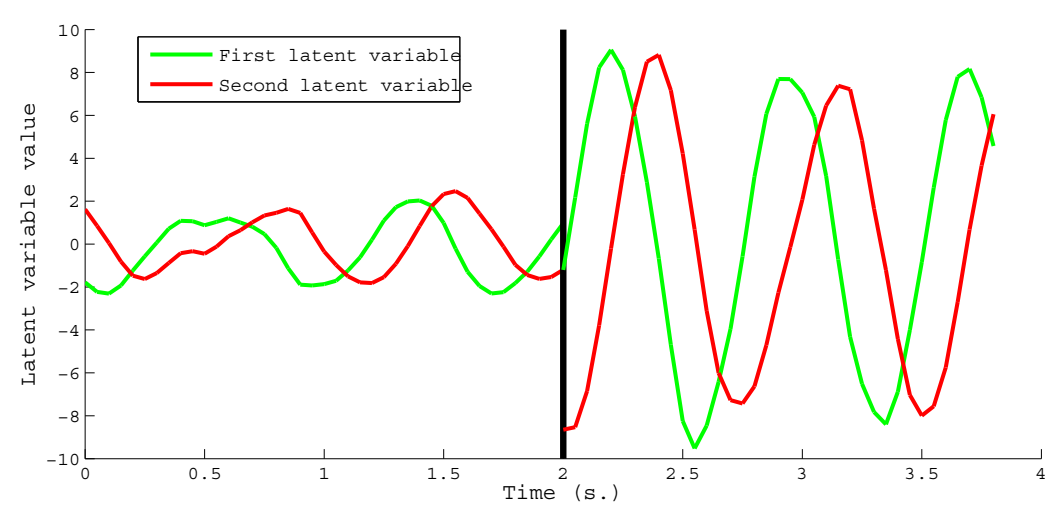

(b) First Latent Variable

Fig. 6. Original and latent variables during both slow and fast gait

relevant ones. Fig. 6 shows an example of both, the original module for the acceleration and the first and second more informative latent variables during a gait episode, which includes fast and slow walking dynamics. Vertical line discriminates walking velocity during a gait episode, the first cycle corresponding to a gait velocity of $35 \mathrm{~cm} / \mathrm{s}$, while the second one represents a magnitude of $189 \mathrm{~cm} / \mathrm{s}$.

It can be observed how latent variables behave similarly to a sinusoidal signal. A direct relationship exists between amplitude of the new signal and human gait velocity. Therefore, the problem of estimating the stride length and velocity could be solved by using regression on latent variables. As depicted in Fig.6(b), both latent variables would discriminate a slow gait episode from a fast one, therefore, for the estimation of step length and velocity both variables $\left(\mathbf{y}_{1}, \mathbf{y}_{2}\right)$ are chosen as input space for the regressor. First two latent variables contain main information described as covariance, as Fig. 8 presents. Only these two latent variables are out of the noise level. 


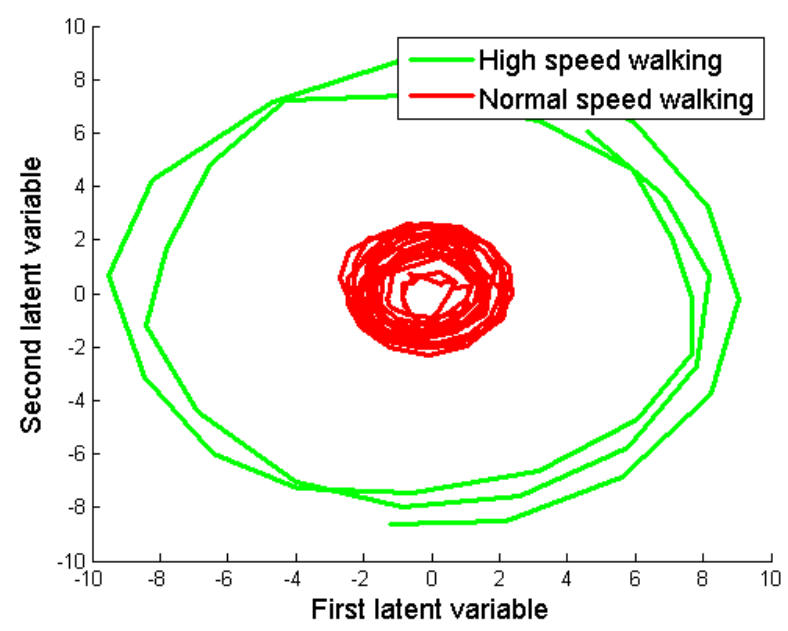

Fig. 7. Recurrence plot of a volunteer

In fact, final features used for the regression are given by

$$
\bar{r}_{4}=\max _{j}\left(w_{j} \cdot \tau\right)
$$

where

$$
w_{j}=\sqrt{\left(y_{j, 1}\right)^{2}+\left(y_{j, 2}\right)^{2}},
$$

and $\tau$ represents the step's duration.

The sum of the square components in (9) represents the instantaneous radius of the trajectory in the space formed by the first two latent variables. A graphical representation is presented in Fig. 7, where two different gait velocities are described.

To the best of our knowledge, it does not exist any method to determine the correct reconstruction of the state space of a DS. Taken's theorem is valid only for noiseless measures although it has been extensively and successfully applied for noisy cases. In this work, since we are measuring gait properties, recurrence plots may clarify whether our reconstruction is valid. Recurrence plots are a common technique helpful to visualize the recurrences of dynamical systems [26]. Given a sequence of (reconstructed) states $x_{1}, \ldots, x_{n}$ of a system, a matrix $M_{n \times n}$ is considered where each element $m_{i j}$ may have two values: 1 when $x_{i} \approx x_{j}$ and 0 otherwise. Note that similarity is defined by $\epsilon$-insensitivity. This matrix is plotted, and periodic motions are reflected by long and uninterrupted diagonals. The vertical distance between these lines corresponds to the period of the oscillation. Figure 9 shows the recurrence plot for a volunteer when using embedding dimension 30 and $\epsilon=10.5$. Similar results were obtained by the rest of volunteers. Periodic orbits are clearly distinguishable, which 


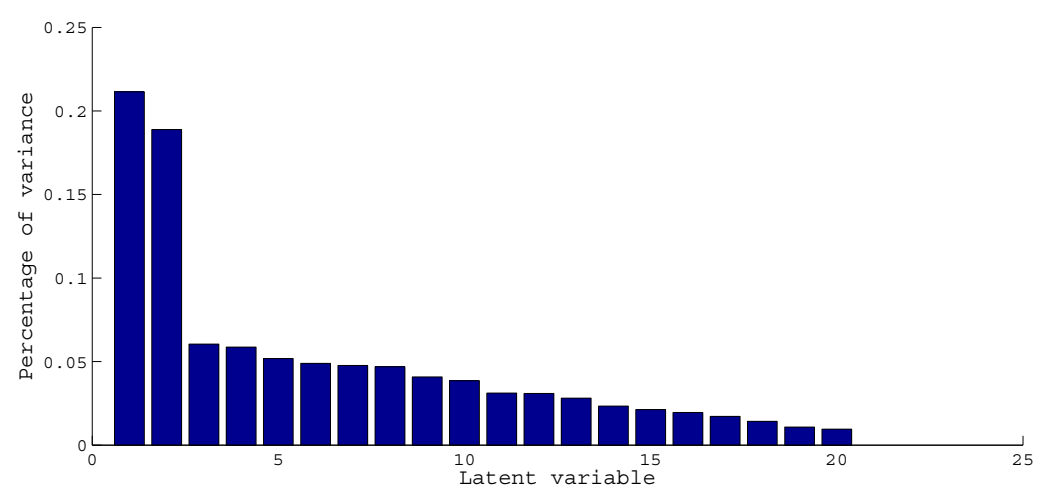

Fig. 8. Variance explained by each latent variable

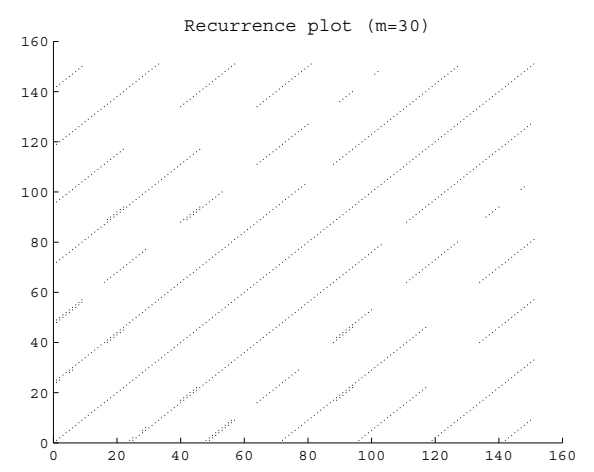

Fig. 9. Recurrence plot of a volunteer

\subsection{Results using Latent Variables}

The method presented was applied on real data obtained from the same experiment in the precedent case, for comparison purposes. This section compares the estimation results of spatiotemporal parameters using $r_{4}$ and when signal characteristics $\bar{r}_{1}, \bar{r}_{2}, \bar{r}_{3}$ from the precedent experiment are used.

Table 4 shows the best results obtained when predicting length and velocity for each regression model $(m=30$, sampling at $50 \mathrm{~Hz})$. In order to evaluate the prediction capability of the the regression using raw data, a randomly generated set ( $80 \%$ of the samples) was used to train $\epsilon$-SVR model, the remaining samples were used to establish the MSE error rate. This process was repeated one hundred times to obtain significant values. A 10-fold cross validation was performed in order to obtain prediction error measures on features extracted from latent variables. This process was repeated 30 times to establish significant values. For the embedding approach, the best result was obtained when 
using $\epsilon$-SV regression with a RBF kernel for both spatiotemporal parameters, which improves the results provided by other type of regressions, (e.g., polynomial or linear kernels). When using those features extracted from raw data, the best result was that provided by the $\epsilon$-SV regression, using a cubic polynomial kernel. From the medical point of view these results may be accurately enough to value the subjects' gait performance.

\begin{tabular}{c|cc}
\hline & Step velocity & Step length \\
\hline Regression & Mean MSE $(\mathrm{cm} / \mathrm{s})$ & Mean MSE $(\mathrm{cm})$ \\
\hline Direct $(\epsilon-$ SVR cubic kernel $)$ & 214.37 & 340.9 \\
Embedding $(\epsilon-$ SVR RBF kernel $)$ & 110.2 & 240.2
\end{tabular}

Table 4

Summary of results for step velocity and step length.

A more understandable prediction error measures of the results are listed in Table 5 and 6 , where a specific estimation and its errors are presented. The relative error between the RMSE and the average value of each spatioxstemporal parameters is presented.

\subsection{Discussion}

Table 4 compares results from embedding approach against those obtained using a direct model, which are also presented in Table 4.3. Time embedding approach outperforms the direct model results. A single value calculated from the reduced reconstruction space outperforms predictions based on different features of the original signal. Figure 6 suggests that it would be easier to extract information from latent variables, and estimation results evidence it.

Estimation errors are presented in Table 5 and 6 . The embedding approach shows better performance on estimating both spatiotemporal parameters. Relative errors between RMSE and the average value are also shown. Embedding approach provides an error of $15.3 \%$ for step velocity and $18.6 \%$ for step length. This apparently high error may not be a problem in most medical applications, since step length and velocity assessment does not require an extremely precise measurements. A long ambulatory assessment of the step length and velocity of patients can be performed through this estimation method, since significative changes will be reflected on the estimations.

A previous study which used 4 gyroscopes reported a RMSE of $7 \mathrm{~cm} / \mathrm{s}$ for step velocity and $8 \mathrm{~cm}$ for step length [8]. Sensors should be located carefully in thigh and shanks, and they were sensitive to orientation. Such results are more accurate than those obtained by the embedding approach used in this study. However, it must be taken into account that this lower precision is a 
Table 5

Velocity Error Case Analysis (Average Velocity $=68 \mathrm{~cm} / \mathrm{s}$ )

\begin{tabular}{c|c}
\hline Approach & Vel Error $(\mathbf{c m} / \mathbf{s})-(\%)$ \\
\hline Embedding $(\epsilon$-SVR RBF kernel) & $10.50-(15.3 \%)$ \\
Direct $(\epsilon-$-SVR 3-degree polynom. kernel) & $14.64-(21.3 \%)$ \\
\hline
\end{tabular}

Table 6

Length Error Case Analysis (Average Length $=83.24 \mathrm{~cm}$ )

\begin{tabular}{c|c}
\hline Approach & Length Error $(\mathbf{c m})-(\%)$ \\
\hline Embedding $(\epsilon$-SVR RBF kernel) & $15.49-(18.6 \%)$ \\
Direct $(\epsilon$-SVR 3-degree polynom. kernel) & $18.46-(22.18 \%)$ \\
\hline
\end{tabular}

consequence of usability restrictions. An unique wearable sensor attached to the waist, which must be insensible to orientation provides quite different and irregular signals between volunteers. Thus, embedding approach has been able to adapt to such signals but resulting in higher errors.

Using the reconstruction of the state space of human gait rather than employing signal features is showed to be more precise for the estimation of gait parameters. Nevertheless, it is possible that other features could obtain more accurate estimations. In order to find them, a large process comprising feature definition, feature selection and testing should be done. By contrast, state space reconstruction combined with PCA analysis has provided a gait representation easily interpretable which made feature definition obvious.

Step velocity estimations are more precise than those obtained for length. The reason may be caused by the nature of the sensor measurements which are accelerations. A measurement error induced by noise may affect length regression stronger than the case of velocity since there is a double cumulative relation which is likely to disturb.

\section{Conclusions}

Three approaches for detecting posture and activities and estimating spatiotemporal parameters of gait have been presented. A common structure based on feature extraction and kernel method is used. Posture changes and activities are detected by SVM classification over features obtained from the measurements of the $9 \times 2$ device. Thus, the device is comparable to some commercially available sensors. Moreover, spatiotemporal parameters of gait are estimated using either a direct model, which extracts features directly from the accelerations comprising a step, or by features extracted from a reconstructed space when the behavior is considered as a DS. 
A new approach for gait parameters estimation is presented, based on the implicit modeling of the human gait as a DS. The state space reconstruction analyzed using PCA is shown to be a simple method to extract gait properties. The goodness of the reconstruction is empirically demonstrated. Human gait is a cyclical process and recurrence plots evidenced that trajectories in the state space reconstruction are also periodic. Furthermore, the trajectories in the latent space are also periodic since the first two variables are a pair of sinusoidal signals.

The feature selection algorithm combined with kernel methods has provided perfect discrimination between activities. Furthermore, kernel methods for regression purposes have resulted in an accurate estimation of spatiotemporal parameters of gait. Using the reconstruction space as input space for the PCA analysis provides an efficient method, obtaining truthful estimations when extracting the most interesting information that is spread along the dimensions of the reconstruction space.

This method provides a comfortable way to extract gait parameters by using a unique sensor located at the waist lateral side. The approach used is insensitive to orientation and assumes changes in the exact location of the sensor between users. Results show slightly lower accuracies in comparison to other systems that use more sensors and are sensitive to the exact position of the devices. It seems that there exists a trade-off between comfortability and precision in inertial sensors for the estimation of gait parameters.

The approaches presented are susceptible to be used for medical purposes. A long term ambulatory assessment of postures, activities and gait parameters is feasible by using the presented algorithms. However, it is considered that a main requirement should be accomplished: usability restrictions imposes a single device so that it may be used during daily life. As algorithms should be processed in the device, extracted information must be saved in order to be analyzed by physicians, future directions are on developing real-time versions of the algorithms presented.

\section{References}

[1] L. Rochester, A. Nieuwboer, K. Baker, V. Hetherington, M. Willems, F. Chavret, G. Kwakkel, E. Van Wegen, I. Lim, D. Jones, The attentional cost of external rhythmical cues and their impact on gait in parkinsons disease: effect of cue modality and task complexity, Journal of Neural Transmission 114 (10) (2007) 1243-1248. doi:10.1007/s00702-007-0756-y.

[2] J. Petrofsky, S. Lee, S. Bweir, Gait characteristics in people with type 2 diabetes mellitus, European Journal of Applied Physiology 93 (5-6) (2005) 640-647. 
doi:10.1007/s00421-004-1246-7.

[3] M. E. Rogers, N. L. Rogers, N. Takeshima, M. M. Islam, Methods to assess and improve the physical parameters associate with fall risk in older adults, Preventive Medicine 36 (3) (2003) 255-264. doi:10.1016/S0091-7435(02)000282.

[4] K. E. Webster, J. E. Wittwer, J. A. Feller, Validity of the gaitrite walkway system for the measurement of averaged and individual step parameters of gait, Gait \& Posture 22 (4) (2005) 317-21. doi:10.1016/j.gaitpost.2004.10.005.

[5] A. Tromp, Fall-risk screening test: A prospective study on predictors for falls in community-dwelling elderly, Journal of Clinical Epidemiology 54 (8) (2001) 837-844. doi:10.1016/S0895-4356(01)00349-3.

[6] W. Zijlstra, A. L. Hof, Assessment of spatio-temporal gait parameters from trunk accelerations during human walking, Gait \& Posture 18 (2) (2003) 1-10. doi:10.1016/S0966-6362(02)00190-X.

[7] A. Salarian, H. Russmann, F. J. G. Vingerhoets, C. Dehollain, Y. Blanc, P. R. Burkhard, K. Aminian, Gait assessment in parkinson's disease: Toward an ambulatory system for long-term monitoring, IEEE Transactions on Biomedical Engineering 51 (8) (2004) 1434-1443. doi:10.1109/TBME.2004.827933.

[8] K. Aminian, B. Najafi, Capturing human motion using body-fixed sensors: Outdoor measurement and clinical applications, Computer Animation and Virtual Worlds 15 (2) (2004) 79-94. doi:http://dx.doi.org/10.1002/cav.v15:2.

[9] S. Lord, L. Rochester, K. Baker, A. Nieuwboer, Concurrent validity of accelerometry to measure gait in parkinsons disease, Gait \& Posture 27 (2) (2008) 357-359. doi:10.1016/j.gaitpost.2007.04.001.

[10] A. Meichtry, J. Romkes, C. Gobelet, R. Brunner, R. Müller, Criterion validity of $3 \mathrm{~d}$ trunk accelerations to assess external work and power in able-bodied gai, Gait \& Posture 25 (1) (2007) 25-32. doi:10.1016/j.gaitpost.2005.12.016.

[11] M. Brandes, W. Zijlstra, S. Heikens, R. van Lummel, D. Rosenbaum, Accelerometry based assessment of gait parameters in children, Gait \& Posture 24 (4) (2006) 482-486. doi:10.1016/j.gaitpost.2005.12.006.

[12] N. Bidargaddi, A. Sarela, L. Klingbeil, M. Karunanithi, Detecting walking activity in cardiac rehabilitation by using accelerometer, 2007, pp. 555-560. doi:10.1109/ISSNIP.2007.4496903.

[13] D. Giansanti, Does centripetal acceleration affect trunk flexion monitoring by means of accelerometers?, Physiological Measurement 27 (10) (2006) 999-1008. doi:doi:10.1088/0967-3334/27/10/006.

[14] C. Bouten, K. Koekkoek, M. Verduin, R. Kodde, J. Janssen, A triaxial accelerometer and portable data processing unit for the assessment of daily physical activity, IEEE Transactions on Biomedical Engineering 44 (3) (1997) 136-147. doi:10.1109/10.554760. 
[15] R. Begg, M. Palaniswami, B. Owen, Support vector machines for automated gait classification, IEEE Transactions on Biomedical Engineering 52 (5) (2005) 828-838. doi:10.1109/TBME.2005.845241.

[16] I. Guyon, A. Elisseeff, An introduction to variable and feature selection, Journal of Machine Learning Research 3 (2003) 1157-1182.

[17] S.-W. Lee, K. Mase, K. Kogure, Detection of spatio-temporal gait parameters by using wearable motion sensors, IEEE Conference on Engineering in Medicine and Biology Society 7 (2005) 6836-96839.

[18] K. Aminian, B. Najafi, C. Büla, P. F. Leyvraz, P. Robert, Spatio-temporal parameters of gait measured by an ambulatory system using miniature gyroscopes, Journal of Biomechanics.

[19] E. Antonsson, R. Mann, The frequency content of gait, Journal of Biomechanics (1981) 39-47.

[20] A. J. Smola, B. Schölkopf, A tutorial on support vector regression, Statistics and Computing 14 (3) (2004) 199-222. doi:10.1023/B:STCO.0000035301.49549.88.

[21] J. Frank, Learning state space models from time series data, in: Multidisciplinary Symposium on Reinforcement Learning, Montreal, Canada, 2009 .

[22] A. Galka, Topics in Nonlinear Time Series Analysis: with implications for EEG Analysis, World Scientific, 2000.

[23] F. Takens, Detecting strange attractors in turbulence, Dynamical Systems and Turbulence (1981) 366-381doi:10.1007/BFb0091924.

[24] Kuan Zhang, Patricia Werner, Ming Sun, F. Xavier Pi-Sunyer and Carol N. Boozer. Measurement of Human Daily Physical Activity. journal of Obesity Research, (11) 1, (2003)

[25] Alejandro Rodrguez-Molinero, Carlos Prez, Albert Sam, Jaume Romagosa. Experimental procedure for motion detection and physical activity data collection with IMU-based body sensors. Technical Report from Universitat Politecnica de Catalunya (UPC-Barcelona TECH), ESAII Department. ESAIIRR-11-01

[26] Norbert Marwan, M. Carmen Romano, Marco Thiel and Jrgen Kurths. Recurrence plots for the analysis of complex systems. Physics Reports, Volume 438, Issues 5-6, January 2007, pp 237-329.

[27] Jan Rueterbories, Erika G. Spaich, Birgit Larsen and Ole K. Andersen. Methods for gait event detection and analysis in ambulatory systems Medical Engineering \& Physics,32 (6) 2010, pp 545-552.

[28] Stephen J Preece, John Y Goulermas, Laurence P J Kenney, Dave Howard, Kenneth Meijer and Robin Crompton. Activity identification using body-mounted sensorsa review of classification techniques. Physiological Measurement 30(4). 
[29] Karol J. ODonovan, Barry R. Greene, Denise McGrath, Ross ONeill, Adrian Burns, Brian Caulfield. SHIMMER: A new tool for temporal Gait analysis. IEEE EMBS Minneapolis, Minnesota, USA, September 2-6, 2009

[30] Lei Wang. Feature Selection with Kernel Class Separability, IEEE Transactions on Pattern Analysis and Machine Intelligence, vol. 30, no. 9, pp. 1534-1546, Sept. 2008

[31] Andrea Giovanni Cutti, Alberto Ferrari, Pietro Garofalo, Michele Raggi, Angelo Cappello, Adriano Ferrari. 'Outwalk': a protocol for clinical gait analysis based on inertial and magnetic sensors. Med Biol Eng Comput (2010) 48:1725

[32] Vincent T. van Hees,Rob C. van Lummel and Klaas R. Westerterp.Estimating Activity-related Energy Expenditure Under Sedentary Conditions Using a Triaxial Seismic Accelerometer. Obesity (Silver Spring). 2009 Jun;17(6):1287-92

[33] Busse ME, van Deursen RW, Wiles CM. Real-life step and activity measurement: reliability and validity. J Med Eng Technol. 2009;33(1):33-41

[34] Kuan Zhang, Patricia Werner, Ming Sun, F. Xavier Pi-Sunyer and Carol N. Boozer. Measurement of Human Daily Physical Activity. Obesity Research (2003) 11, pp. 3340

[35] Mazzocca et al.: Arthroscopic Single-Row Versus Double-Row Suture Anchor Rotator. Cuff Repair, American Journal of Sports Medicine, Vol. 33, December 2005 